\title{
Development of Dispatching Automation Time Synchronization Management Module Based on VxWorks Embedded Device
}

\author{
Chun-Bing JIANG ${ }^{\mathrm{a}}$ \\ 2 Beinong Road, Huilongguan Town, Changping District, Beijing, China \\ ajcb808@163.com
}

Keywords: Time Synchronization Management Module, Vxworks, Embedded System.

\begin{abstract}
This paper takes "The research and application of scheduling clock synchronization system" science project of Jilin province electric power company control center. This paper develops a time synchronization management module based on VxWorks embedded system in the end station dispatching automation clock synchronization system. The network shutdown and the measurement and control device used in the original plant is based on the VxWorks embedded system, which cannot meet the requirements of time synchronization management. The management module mentioned above solves the tough problem that the non-open source embedded device cannot.
\end{abstract}

\section{Introduction}

The power system can be said to be a system associated with the time. Current, voltage, angle and angle change are all waveform based on the time axis. Nowadays, supercritical an ultra supercritical units have already connected with the grid, and regional power grid interconnection and UHV transmission technology are developed at the same time. The safe and stable operation of power grid put forward some new requirements for the electric power automation equipment, especially for the time synchronization requirements of relay protection device, automatic device, security and stability control system, energy management system (EMS) and the production information management system. These devices and systems should be operated base on the unified time standard in order to meet the require of time consistency on the sequence of events (SOE), fault recorder and real time data acquisition, and to make sure that the accuracy of line fault location, the phase and angle dynamic monitoring, the generator and system parameter calibration and the fault analysis are on a high level, so that to improve the efficiency and reliability of the operation[1].

However, as the rapid development of the smart grid, the current scheduling automation time synchronization exists many urgent technical problems. For example, some factory station ends have more than one set of clock source, some stations have problem of low accuracy time synchronization like pulse time synchronization and network time synchronization, and some scheduling station ends can't realize online monitoring and condition assessment the equipments timing and to be timing. When there's a fault or an accident comes to the power system, it is difficult to analyze and judge the cause of the accident because of the confusion of the time scale of the uploaded information, what's worse, the center of load control may send wrong commands.[2] Aiming at the present safety problem of time synchronization of the power system, we need to do some research to analyze the project of time synchronization in the respect of clock source, time synchronization, synchronization of physical connection, the safety protection of the secondary system in power system and the timekeeping of timing equipments, and propose some corresponding management strategies.

To solve the problems mentioned above, this paper dose some research about the time synchronization management of the inner dispatching center, the inner substation and the things between them. The research of automatic dispatching time synchronization mainly including its partition scheme design according to the function area and its implementation of the scene, developing a scheduling station time synchronization management module that is easy to deployment and implement on the D5000 platform. Also, this paper develops a set of automatic 
dispatching time synchronization management system base on the time synchronization management module of VxWorks embedded device. Meanwhile, this paper realizes the state assessment and fault location across check algorithm in dispatching time synchronization system.

\section{The Structure and Management Strategies of Dispatching Automation Time Synchronization System}

This paper divides dispatching automation time synchronization system into three parts according to its function area, including the internal of the substation end, the internal of the dispatching center and the part between substation and center. [3]The system function division and the overall program design are as shown in Fig. 1 and Fig. 2.
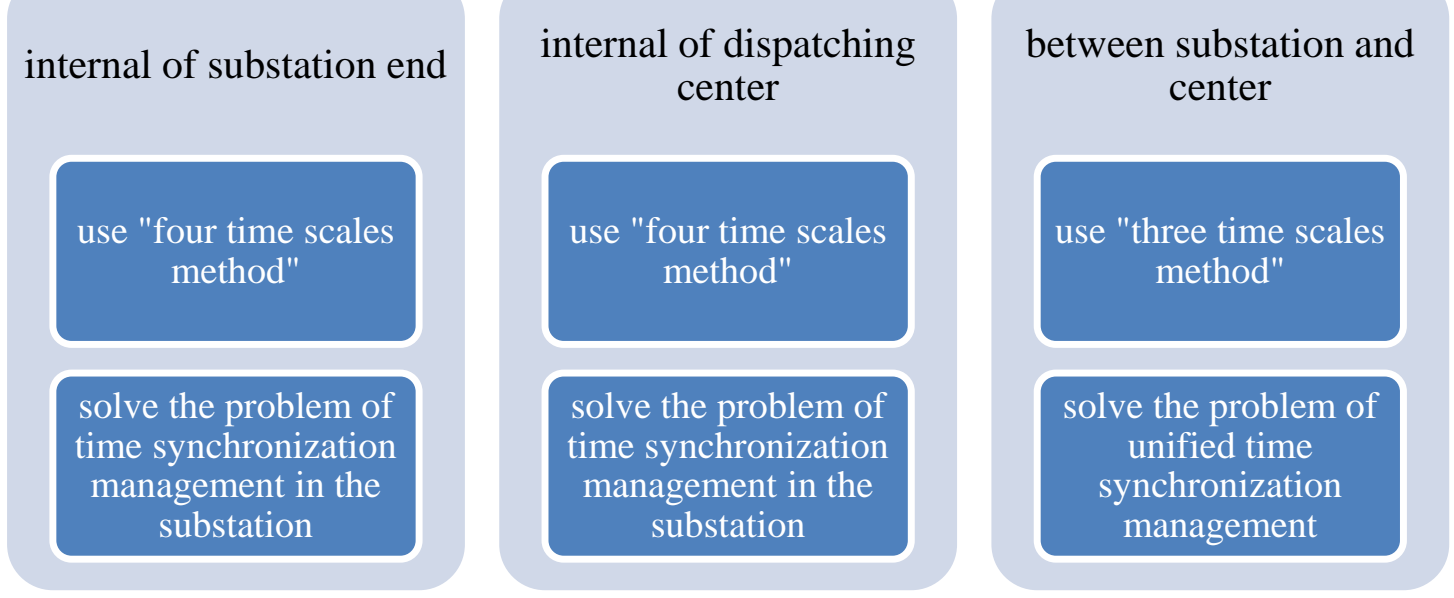

Fig. 1. Time synchronization system function area division

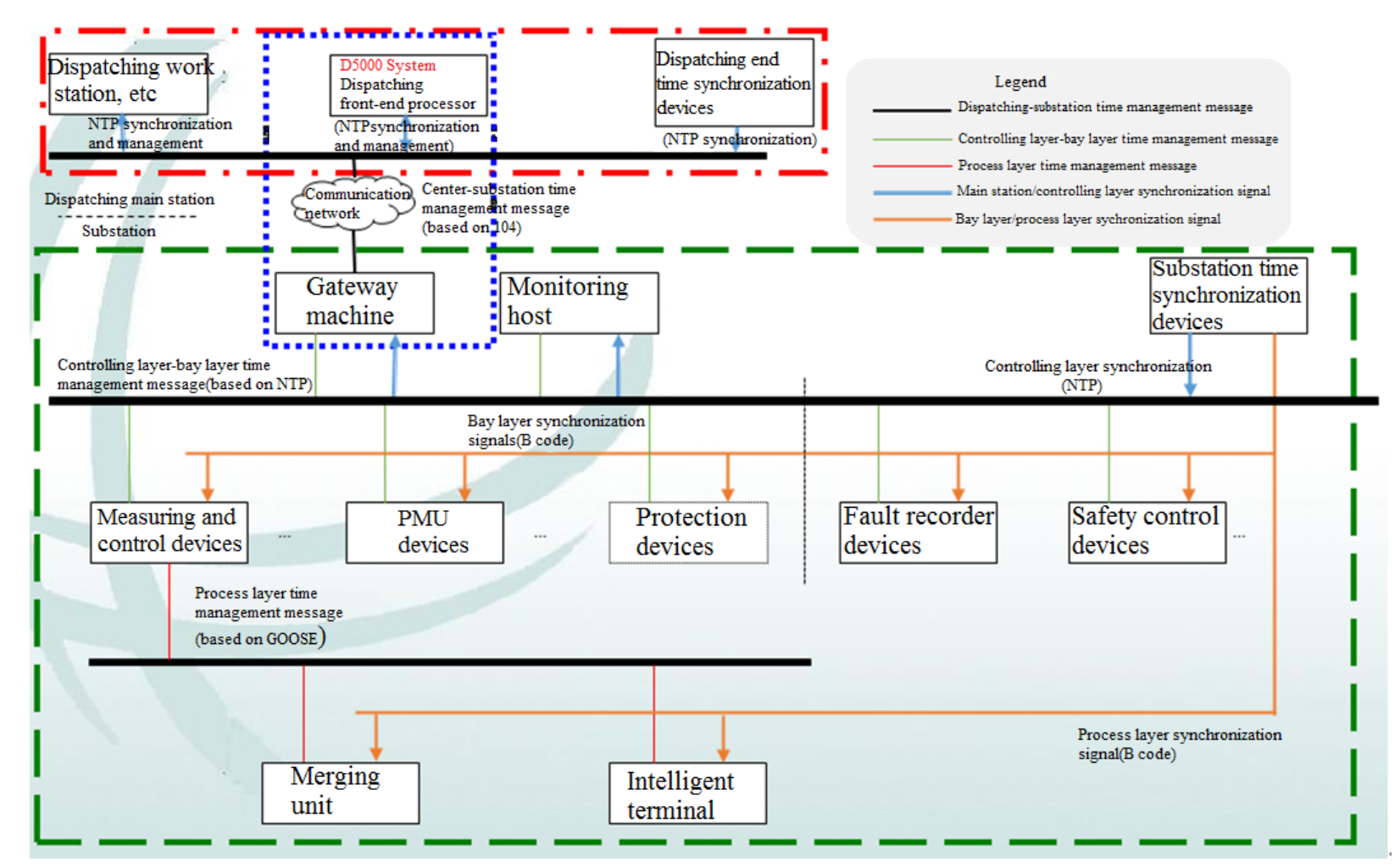

Fig. 2. Time synchronization system program design

\section{Closed-loop Principle}

Time synchronization closed-loop management refers to that time synchronization system consists of time servicing end and time serviced end. Synchronize status online monitoring objects contain 
time servicing end and time serviced end. The clock and time setting devices can be made up into closed loop so that time setting state can be monitored and monitoring results can be handed up. The time synchronization system can be included into automatic monitoring system management. It is shown in Fig.3.

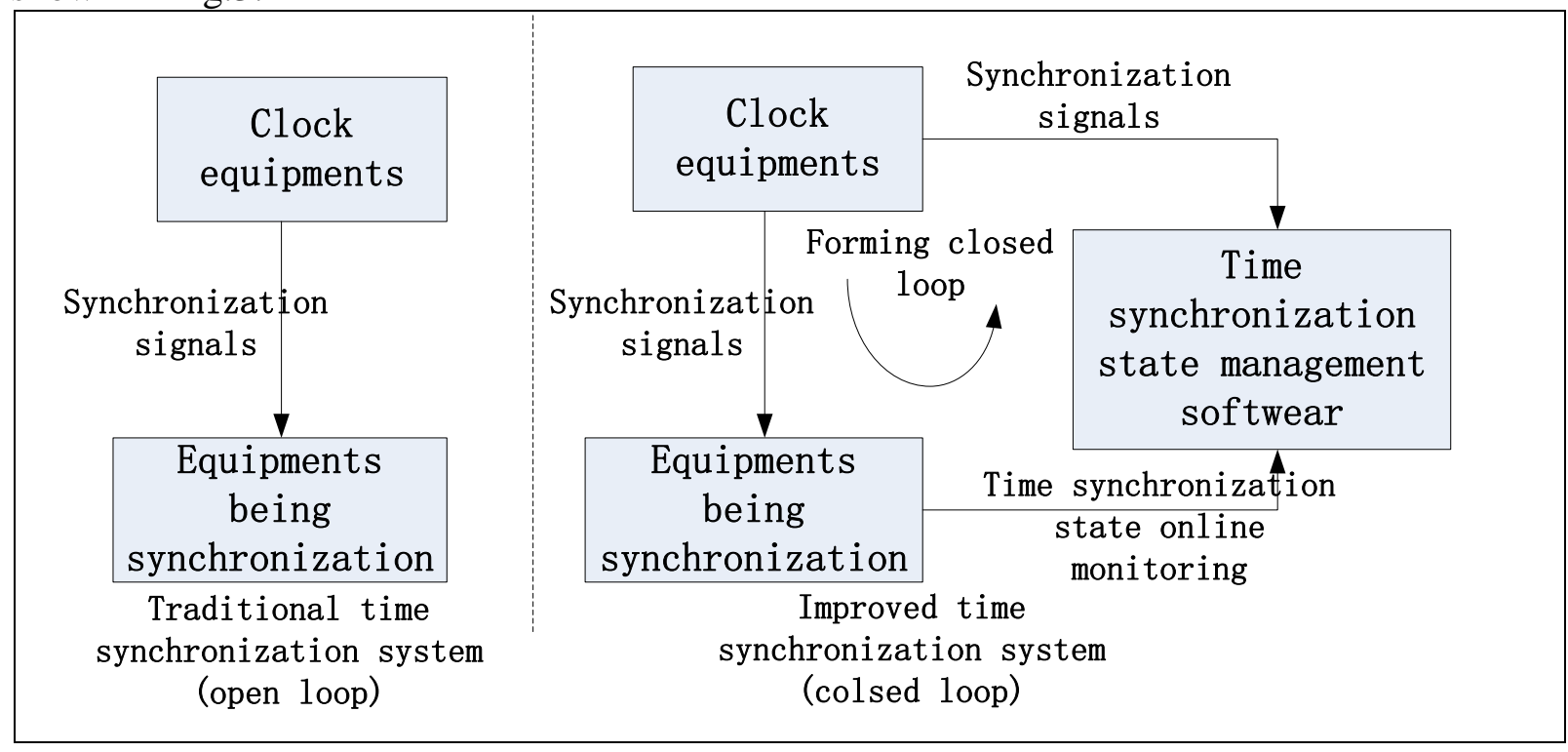

Fig. 3. The principle of time synchronization management open and closed loop

The data of time synchronization state online monitoring comes from two parts: the synchronization state measurement data and the equipment status self-checking data. The equipment status self-checking is mainly a strategy used to detect the fault point of the device being monitoring, which is set based on the predictable fault. The synchronization state measurement is to detect the results come from the faults which the equipments being monitoring cannot foresee by themselves from the outside. The two methods moderately ensure the performance and reliability of the time synchronization monitoring[4].

\section{Ping-pong principle with Four Time Scales Method}

The four time scales ping-pong principle is mostly used in the internal of the dispatching main station and the internal of the substation (the station control-interval layer, the station layer monitoring host and the other equipments of the substation).

As time synchronization management end, station monitoring host monitors and manages the time synchronization statue of time synchronization device, measurement and control device, fault recorder device, PMU ( Power Management Unit ), intelligent terminal, which follows the NTP ( Network Time Protocol) ping-pong principle( four time scales)[5]. Smart grid dispatching technical support system for changing the pre-application aiming at time synchronization monitoring function, pre-application like IEC104 or DL476 protocol have had time synchronized in ping-pong principle with substation automation system, which can check the clock synchronization accuracy of every substation according to the result. Thus, we can make sure the accuracy of time synchronization of the whole network. The specific principle and the time synchronization process are shown in Fig. 4. 


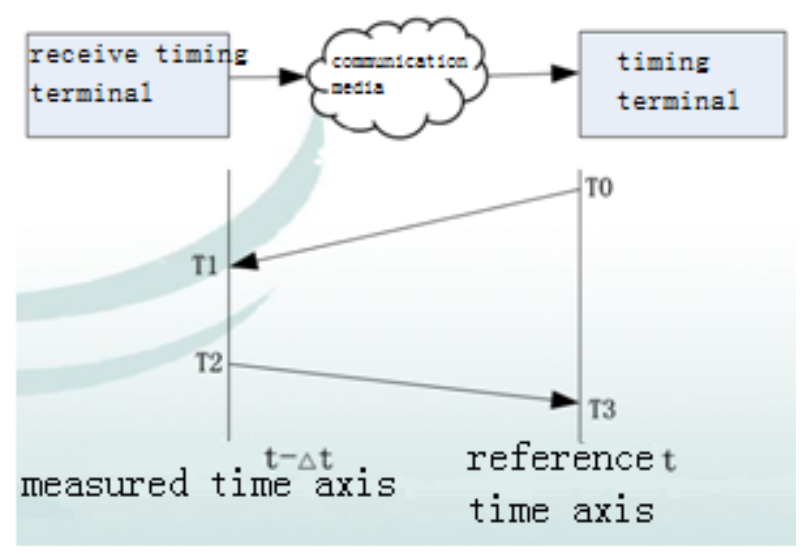

Fig. 4. The four time scales ping-pong principle

In Fig. 4, T0, T1, T2 and T3 are four time scales. $\triangle \mathrm{t}$ is a clock error, the calculation formula is

$$
\triangle \mathrm{t}=[(\mathrm{T} 3-\mathrm{T} 2)+(\mathrm{T} 0-\mathrm{T} 1)] / 2
$$

where T0 is the time scale of the main station to send "measurement clock request", T1 is the time scale of the substation to receive "measurement clock request", T2 is the time scale of the substation to send "the results of measurement clock request" and T3 is the time scale of the main station to receive "the results of measurement clock request".

The dispatching main station has a pre-gateway machine application which sends "measurement clock request" command to the pre-application of the substation automation system regularly. After a " measurement clock request", the computer will calculate the clock error between the main station and the substation according to the value of T0, T1,T2 and T3. If the clock error exceeds the preset system error threshold, it will give an alarm. The pre-gateway application of the dispatching main station will detect the time synchronization device of the dispatching center at the same time, in accordance with the data transmission protocol and alarm format definite by the time synchronization device, and then receive the alarm information from time synchronization device. Meanwhile, according to the ping-pong timing principle mentioned above, the pre-application will also calculate the timing error of the time synchronization device in the dispatching center. If the clock error $\Delta t$ exceeds the preset system error threshold, also, the system will report an alarm. The main station system receives the statue data analysis results of the total substation by IEC104 or DL476, so that it can monitor the real-time timing state information of each system and equipment in the substation.

\section{Three Time Scales Ping-pong Principle}

The time synchronization monitoring of the dispatching main station and the substation both use the three time scales ping-pong principle, as shown in Fig. 5. 


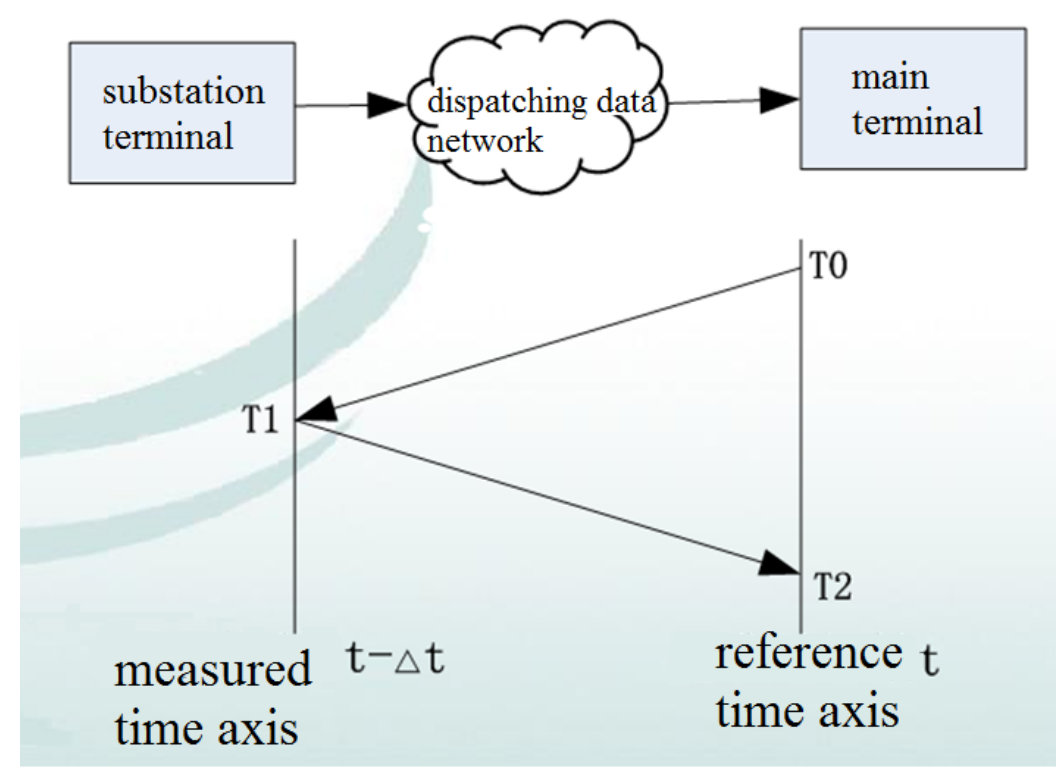

Fig. 5 Three time scales ping-pong principle

In Fig. 5, T0, T1, T2 are three time scales, $\Delta \mathrm{t}$ is the clock error that the main station leads the substation (leading positive, lagging negative). The calculation formula is

$$
\triangle \mathrm{t}=[(\mathrm{T} 2+\mathrm{T} 0) / 2]-\mathrm{T} 1
$$

where T0 is the time scale of the main station to send "measurement clock request", T1 is the time scale of the substation to receive "measurement clock request", T2 is the time scale of the main station to receive "the results of measurement clock request".

The dispatching main station has a front-end processor which sends "measurement clock request" command to the pre-gateway machine of the substation system regularly. After a " measurement clock request", the computer will calculate the clock error between the main station and the substation according to the value of $\mathrm{T} 0, \mathrm{~T} 1$ and $\mathrm{T} 2$. When the front-end processor of the main station roll polling a monitoring value that is out of the threshold, it will continuously monitoring 5 times in a cycle of 1 second and then average the results after removing the extreme values. If the average value is out of the threshold, then the object being monitored is considered that the time synchronization is abnormal, the computer will generate a corresponding alarm information[5].

The front-end processor of the main station updates the communication software (such as104, 476 and other timing commands that is originally in the message) towards the substation monitoring system, so that it can realize the real-time monitoring to the time synchronization state of the substation (data communication gateway). The time synchronization management error between the main station and the substation reaches microsecond level.

\section{NTP Protocol Configuration Principle}

When time synchronization system is monitoring, the NTP adopts client/server pattern. In this pattern, the time management server is the client, and the device being monitoring is the server. The time management server periodically sends message to the device being monitoring. The time management server calculates the clock error according to the clock message returned by the monitored device, but it does not modify the clock of the monitored device.[6] The network time protocol NTP message format is shown in Fig.6. 


\begin{tabular}{|c|c|c|c|c|c|}
\hline 01 & 4 & 7 & 15 & 23 & 31 \\
\hline LI & VN & Mode & Stratum & Poll & Precision \\
\hline \multicolumn{6}{|c|}{ RootDelay (32bits) } \\
\hline \multicolumn{6}{|c|}{ Root dispersion (32bits) } \\
\hline \multicolumn{6}{|c|}{ Reference identifier (32bits) } \\
\hline \multicolumn{6}{|c|}{ Reference timestamp (64bits) } \\
\hline \multicolumn{6}{|c|}{ Originate timestamp (64bits) } \\
\hline \multicolumn{6}{|c|}{ Receive timestamp (64bits) } \\
\hline \multicolumn{6}{|c|}{ Transmit timestamp (64bits) } \\
\hline \multicolumn{6}{|c|}{ Authenticator (optional 96bits) } \\
\hline
\end{tabular}

Fig.6 The network time protocol NTP message format

(1) Reference Identifier syllable, reference time source. According to the NTP standard, it can be extended beyond the predefined tag. The server and the client for monitoring should be filled with "TSSM" (Time Synchronization Status Monitoring). The identification itself is time synchronization monitoring source, so that it can be distinguished from the normal timing NTP server. The monitoring software should not respond to requests outside of the TSSM identity.

(2) Originate Timestamp syllable, the local time of the transmitting terminal when the NTP request message leave the terminal. When the time management server monitoring software(client) requests, the Value which this syllable should fill should be stored in the local memory, and the syllable of the message to be sent should all be filled with 0 , which means that the software will not provide the time reference to the measured object.

\section{The Development of Measurement and Control Device Based on the Embedded VxWorks System}

The traditional gateway machine and measurement-control device of substation are all solidified embedded system based on the VxWorks. There is a large amount of these machines and devices, and cover a wide area, but they can still not meet the requirements of the time synchronization management. Therefore, this paper researches upon embedded VxWorks system, embeds time synchronization program and module from the bottom program properly without influencing the safe and reliable operation of the original system, to meet the technical requirements of the time synchronization management system.

\section{The Introduction of The Embedded VxWorks System}

VxWorks is an embedded real-time operation system. The embedded system is that the users develop and design a board by them own, which usually has a CPU. The VxWorks supports 32 CPU, including x86 of Intel, 68k of Motorola, i960 of PowerPC, MIPS, ARM, Intel and SH of Hitachi. The board users design usually does not have the ability of developing by itself, which means that we need a universal machine to assist with the development. This universal machine can be a PC or a workstation. The universal machine which aids to develop the software is called the host, and the board users develop by themselves is called the target. There should be a integrated development environment (IDE) in the host to assist us develop software. Then environment can be run on Windows95/NT or UNIX, which includes cross compiler and cross debugger. The cross compiler is to compile code IMAGE in the host computer which can be run on the target machine. And the cross debugger is to achieve foreground and background debug through a kind of coupled manner between the host and the target. The IDE on the host is called Tornado, the executable code IMAGE that is compiled in the target machine is called VxWorks. When the system installs, the IDE and the VxWorks raw material (some obj. files) are also installed on the host.[7] 


\section{The Implementation of Communication, Synchronization and Mutex Mechanisms}

In terms of communication, synchronization and mutex mechanism(network communication), VxWorks provides some network tools to complete the message transmission: sockets implement the task message transmission of running in VxWorks systems or other systems; remote procedure call(RPC) allows the task to call a procedure of another host (the operating system should be VxWorks or others). The remote file can use NFS, RSH, FTP,TFTP and other ways to access to host files. It is shown in Fig. 7.

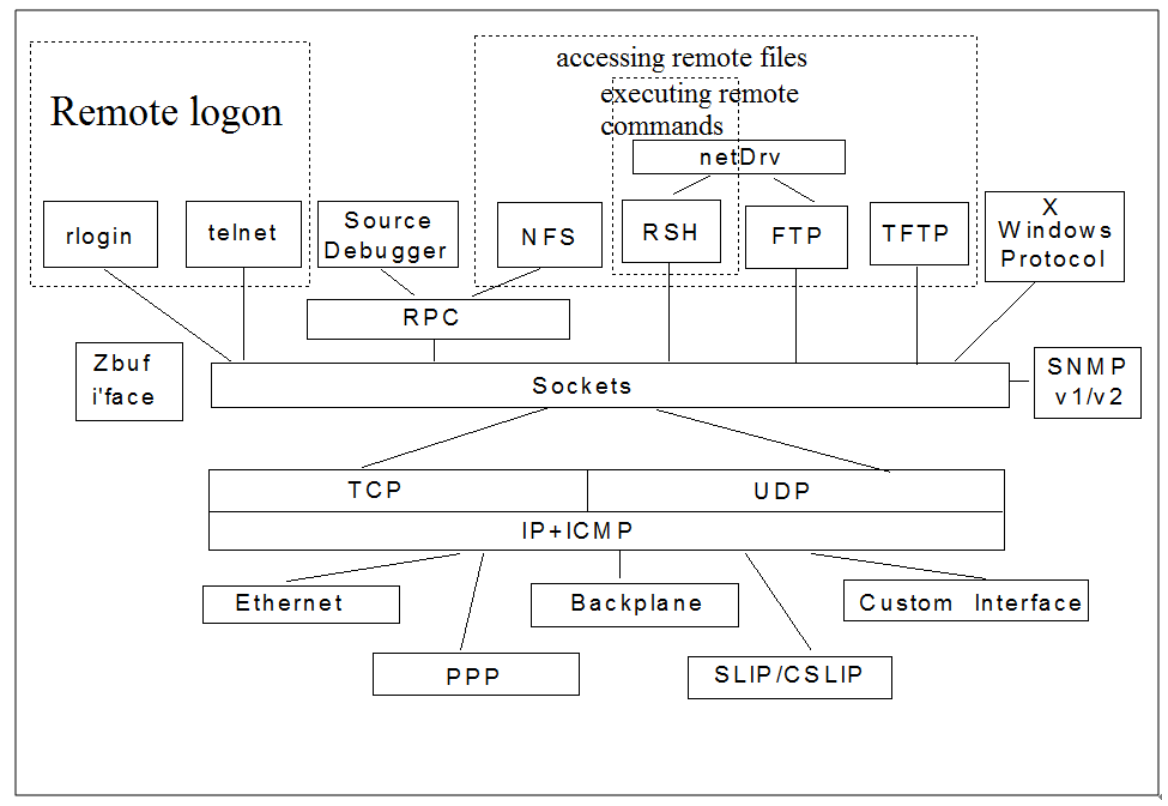

Fig. 7. Communication, synchronization and mutex mechanism(network communication)

VxWorks Kernel interrupt service calling program is as follows

intConnect()

intCount()

intLevelSet()

intLock ()

intUnlock ()

intVecSet()

intVecGet()
Connect the $\mathrm{C}$ function to the interrupt vector;

Get the current interrupt overlay depth;

Set program interrupt level;

Disable interrupt;

Able interrupt;

Set exception vector;

Get exception vector;

\section{The Implementation of Time Management Function}

The time management provides function as follows: maintaining system calendar clocks; timeout processing during the tasks are waiting for the message packets, semaphores, events or memory segments; waking up or sending alerts to a task at a certain time interval or at a specific time; dealing with time slice polling in task scheduling.

Time management calling program is:

tickAnnounce()

tickSet()

tickGet()

timer_create()

timer_gettime()

timer_settime()

timer_connect()

timer_cancel()

sysClkRateSet()
Announce system kernel clock "tick";

Set the kernel clock counter value;

Get the kernel clock counter value;

Create a clock;

Get the current remaining value of the clock's given value;

Set the clock value;

Connect the user function and the clock signal;

Cancel a clock;

Set system clock rate 


\section{Programming Based on Tornado Development Environment}

As an integrated environment, Tornado development environment is an efficient and clear graphical real-time application development platform, and is a development and debugging tool completely embedded-system-oriented. [8]The Tornado development environment is shown as Fig. 8.

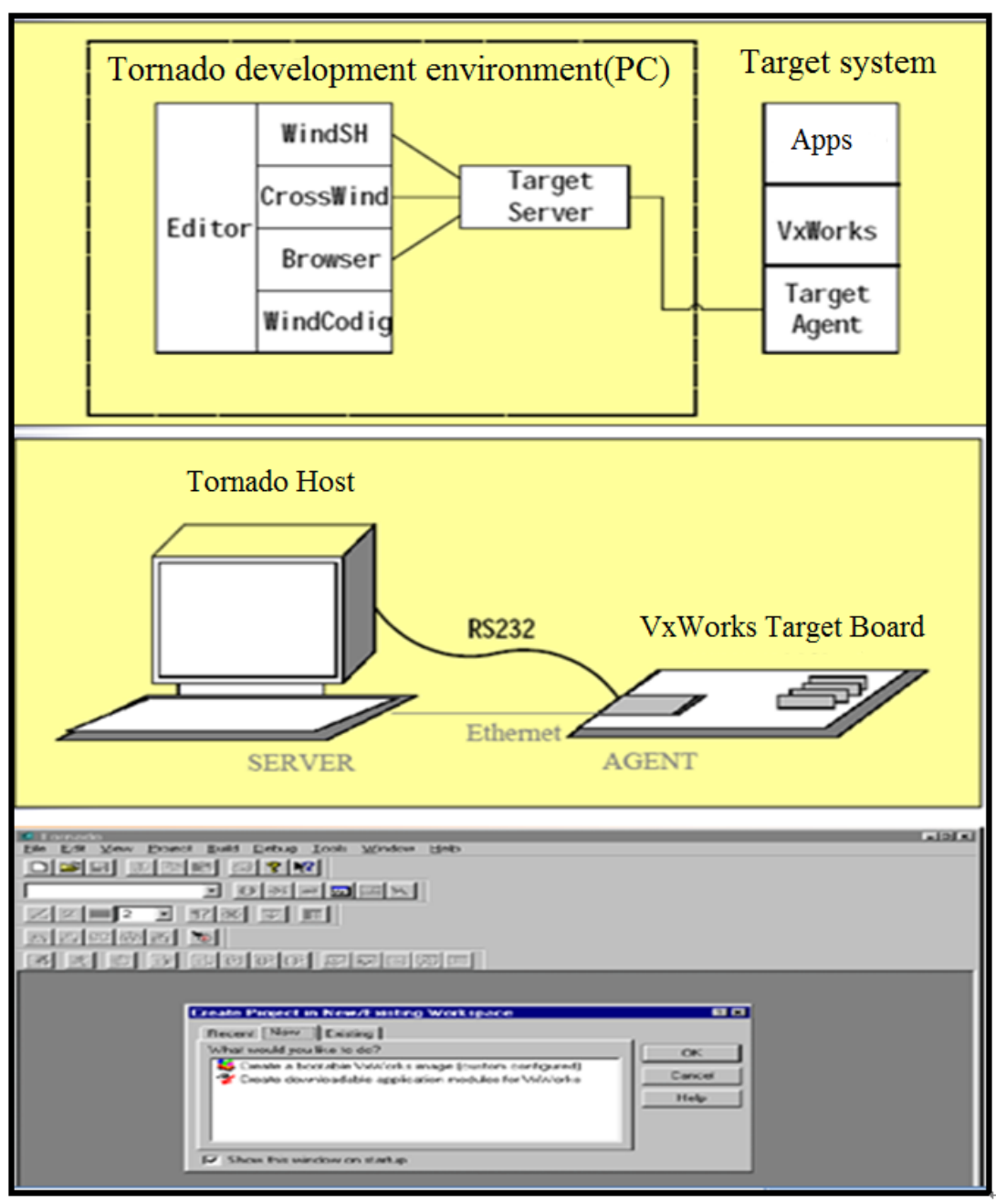

Fig. 8. The Tornado development environment

To create time synchronization management module, the NTP algorithm should be programmed in $\mathrm{C}$ language and then compiled. And the interrupt service sub routine is invoked by the task queue method to write and transmit the time synchronization data.[9] The structure of the module is shown in Fig. 9. 


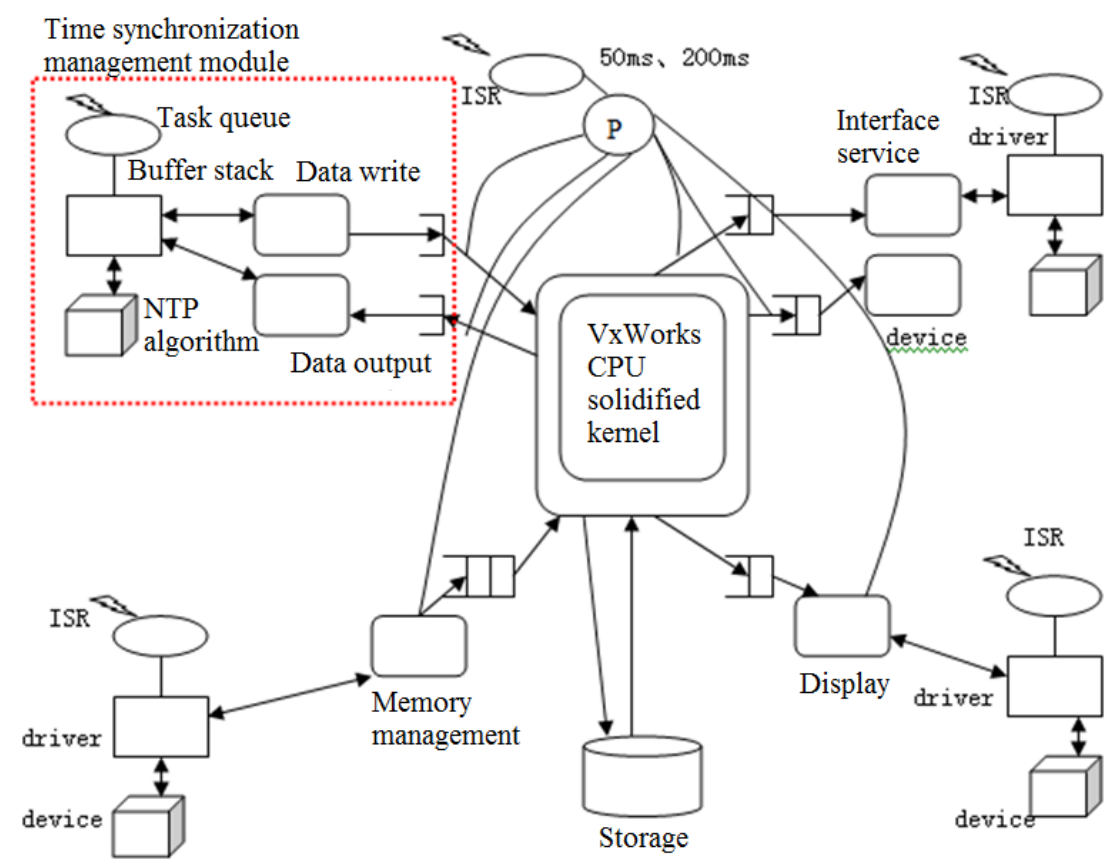

Fig. 9. Time synchronization management module structure

The NTP protocol configuration message mutual flow and frame format are shown in Fig. 10. Time management end Embedded VxWorks system

(a)

Link reset flow Reset remote link(fixed frame)

(b)

Calling time flow

Call data(fixed frame)

Terminal time frame(variable frame)

(c)

Data request frame(fixed frame)

Reset determined frame (single-character frame)

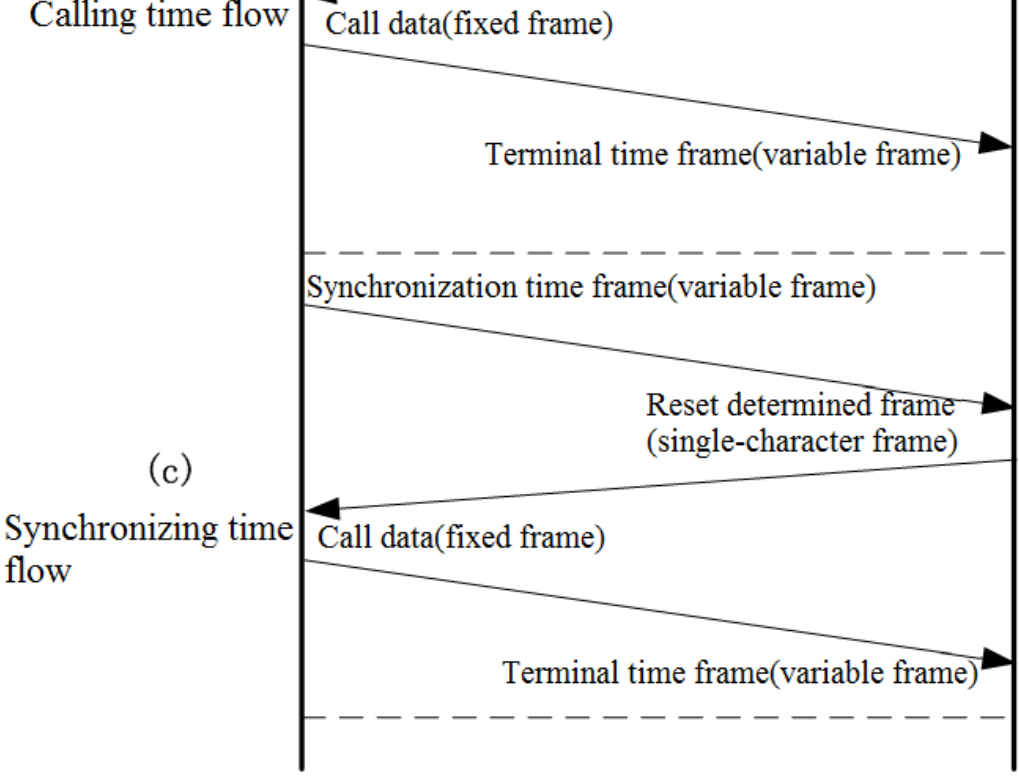

Fig. 10. NTP protocol configuration implementation process flow 
Time synchronization data acquisition and processing are carried out by polling. For the embedded VxWorks measurement and control device, it plays "ping-pong" with the host of the main station control layer, and manage the device and nodes of process layer at the same time. The Fig. 11 describes the data message acquisition and processing process based on time synchronization on-line monitoring and management of power system, and provide a specific implementation method for embedded system engineering applications.

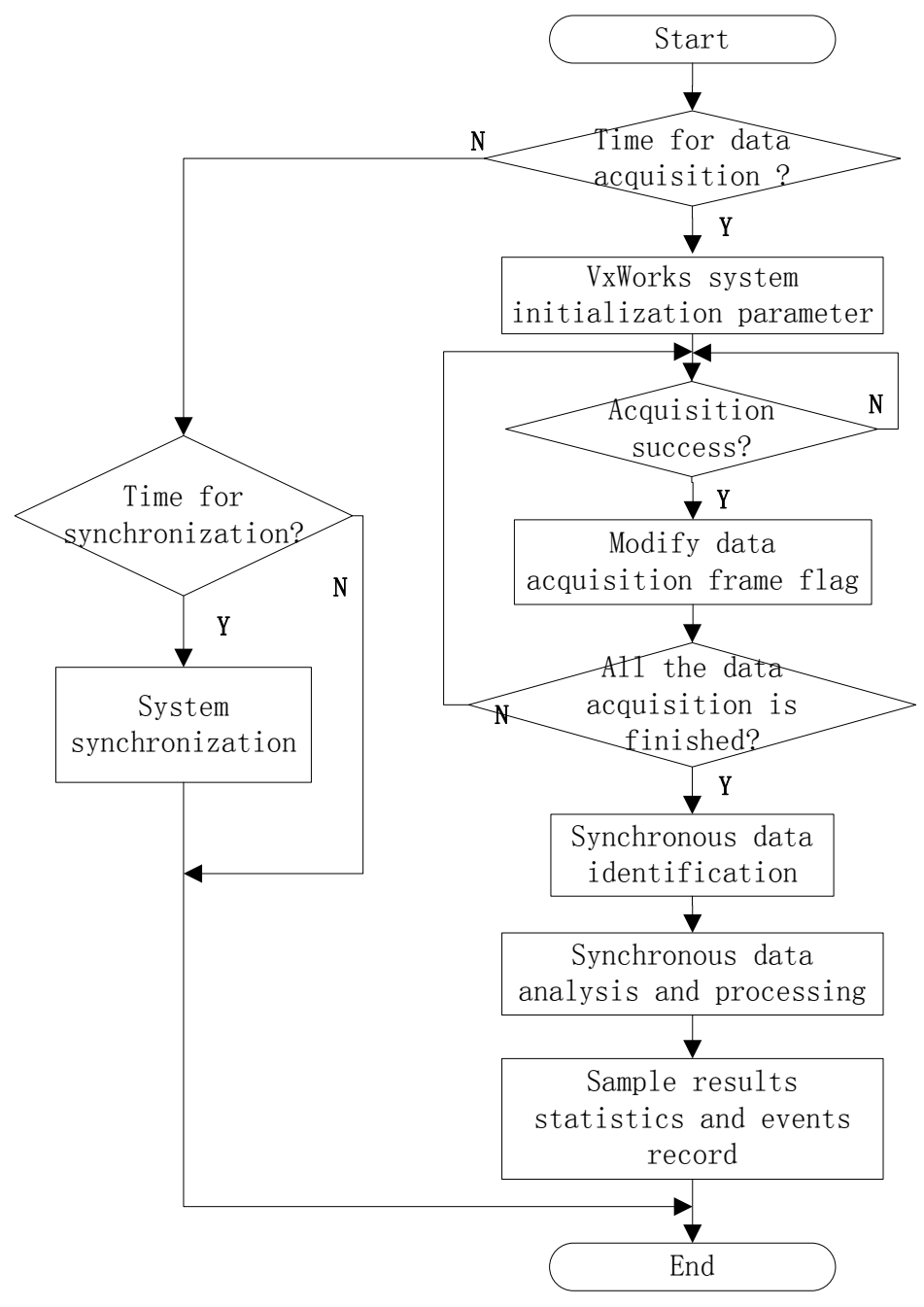

Fig. 11. Time synchronization data acquisition and processing flow

\section{Testing}

The paper time synchronization module this paper proposed has completed the test by the power industry power system automation equipment quality inspection and testing center after the tests in laboratory and the third party. Among them ,the projects like time synchronization status on-line monitoring protocol configuration, detection of synchronization status measurement function, measurement of synchronization deviation, etc, are put forward for the first time and are detected to be qualified, which means the functions of time synchronization module are realized.

\section{Summary}

This paper introduces the structure of the dispatching automation time synchronization system and the principle and method of its management strategies. Then, aiming at the problem that the traditional gateway and measuring-control devices of substation are based on the solidified VxWorks embedded system which cannot meet the requirement of time synchronization, this paper develops a time synchronization management module based on the VxWorks embedded device. 
This module overcomes the problem that the non-open source embedded devices are out of synchronization, and realize the time synchronization management to the original timing equipments. The capture of the problem marks the full access of all the time service equipments and lays the foundation for the management of multi-layer and stereo time synchronization systems.

\section{References}

[1] Zhou G P. Preliminary Study of Clock Synchronization in Power Grid Dispatching Automation System [j]. Guangxi Electric Power Engineering, 1996 (04): 66-68.

[2] Aweya J, Al Sindi N. Role of Time Synchronization in Power System Automation and Smart Grids: IEEE International Conference on Industrial Technology[Z]. 20131392-1397.

[3] Huang J, Hou K, He Y, et al. The Construction of the Clock Synchronization System in Management Information Domain of Power Grid: Power and Energy Engineering Conference[Z]. 20121-5.

[4] Hua M, Dong L. A closed-loop adjusting strategy for wireless HART time synchronization: International Symposium on Communications and Information Technologies[Z]. 2011131-135.

[5] Peng Z Q, Huo X S, Zeng F, et al. Application Analysis of Time Synchronization State On-Line Monitoring Technology for Power System[J]. East China Electric Power. 2014.

[6] Netze H. Network Time Protocol Version 4: Protocol and Algorithms Specification[J]. Heise Zeitschriften Verlag. 2010.

[7] Chen H M, Wang L F, Cheng-Fu W U, et al. Design and Realization of Distributed Measurement System Based on VxWorks[J]. Measurement \& Control Technology. 2009, 28(10): 71-74.

[8] Hole X Y. Embedded Real-Time Operating System VxWorks and its Development Environment Tornado [Z]. China Electric Power Press, 2002.

[9] Acoustics I O. Network Communication based on Buffering Queues in VxWorks[J]. Microcomputer Information. 2009, 25(11): 84-85. 